\title{
INFLUÊNCIA DO SISTEMA DE PLANTIO SOBRE ATRIBUTOS DENDROMÉTRICOS E FAUNA EDÁFICA, EM ÁREA DEGRADADA PELA EXTRAÇÃO DE ARGILA ${ }^{(1)}$
}

\author{
Cristiane Figueira da Silva ${ }^{(2)}$, Marco Antônio Martins ${ }^{(3)}$, Eliane Maria Ribeiro da Silva ${ }^{(4)}$, \\ Marcos Gervasio Pereira ${ }^{(5)}$ \& Maria Elizabeth Fernandes Correia ${ }^{(4)}$
}

\begin{abstract}
RESUMO
O plantio consorciado de eucalipto com leguminosas pode promover a melhoria da qualidade biológica do solo em áreas degradadas e também ser vantajoso para as espécies do consórcio. $O$ objetivo deste trabalho foi avaliar a influência de sistemas de plantios (puros e consorciados) de Acacia mangium (AM), Sesbania virgata (SV) e Eucalyptus camaldulensis (EC), sobre o desenvolvimento das plantas em estudo (variáveis dendrométricas) e especificamente sobre a fauna da serapilheira e dos primeiros $5 \mathrm{~cm}$ do solo. Realizou-se um experimento, cujo delineamento utilizado foi o de blocos casualizados com seis tratamentos e três repetições. Os tratamentos utilizados para avaliação dos atributos dendrométricos foram: 100EC $(100 \% \mathrm{EC})$ e $100 \mathrm{AM}$ (100 \% AM); 50EC:50AM (50 \% EC + 50 \% AM); 50EC:50SV (50 \% EC + 50 \% SV); e 50AM:50SV (50 \% AM + 50 \% SV). Para avaliação da fauna do solo foram: 100EC, 100AM, 100SV (100 \% SV), 50EC:50SV e $50 \mathrm{AM}: 50 \mathrm{SV}$. Aos 48 meses após o plantio, foram feitas medições de altura (H) e diâmetro à altura do peito (DAP) das espécies $E$. camaldulensis e $A$. mangium e estimativas da área basal (AB) e volume de madeira com casca por indivíduo (VCI). Na serapilheira e no solo (0$5 \mathrm{~cm}$ ), foram avaliadas a abundância e diversidade da fauna edáfica. $O E$. camaldulensis quando cultivado em consórcio com as leguminosas apresentou maior DAP, AB e VCI. Em contrapartida, a A. mangium não teve essas variáveis influenciadas quando em consórcio com o E. camaldulensis e com a S. virgata. Plantios de $E$. camaldulensis e $S$. virgata em consórcio promoveram maior abundância total de organismos e maiores valores dos índices de diversidade
\end{abstract}

(1) Parte da Tese de Doutorado da primeira autora, em Produção Vegetal, apresentada à Universidade Estadual do Norte Fluminense Darcy Ribeiro - UENF. Recebido para publicação em 30 de outubro de 2012 e aprovado em 31 de julho de 2013.

(2) Pós-Doutoranda, Instituto de Agronomia, Universidade Federal Rural do Rio de Janeiro - UFRRJ. BR 465 km 7. CEP 23890000 Seropédica (RJ). E-mail: cfigueirasilva@yahoo.com.br

(3) Professor Titular, Centro de Ciências e Tecnologias Agropecuárias - UENF. Av. Alberto Lamego, 2000, Parque Califórnia. CEP 28013-602 Campos dos Goytacazes (RJ). E-mail: marco@uenf.br

(4) Pesquisadora, Embrapa Agrobiologia. BR 465 km 7. CEP 23890-000 Seropédica (RJ). E-mail: eliane.silva@embrapa.br, elizabeth@cnpab.embrapa.br

(5) Professor Associado IV, Instituto de Agronomia, UFRRJ. E-mail:gervasio@ufrrj.br 


\author{
de Shannon e Pielou, principalmente no que se refere ao compartimento \\ serapilheira.
}

Termos de indexação: Eucalyptus camaldulensis, Acacia mangium, dendrometria.

\title{
SUMMARY: INFLUENCE OF PLANTING SYSTEMS ON DENDROMETRIC PROPERTIES AND SOIL FAUNA IN AN AREA DEGRADED BY CLAY EXTRACTION
}

\begin{abstract}
The intercropping of Eucalyptus with legumes can promote an improvement of soil biological quality in degraded areas and also be advantageous for the species of the consortium. The aim of this study was to evaluate the influence of planting systems (monoculture and consortium) of Acacia mangium ( $A M$ ), Sesbania virgata (SV) and Eucalyptus camaldulensis (EC) on the biological characteristics of the plants (dendrometric attributes) and specifically on the fauna in the litter and the surface soil layer $(0-0.05 \mathrm{~m})$. An experiment in a randomized block was conducted with six treatments and three replications. The treatments used to evaluate the dendrometric attributes were: $100 \%$ EC and $100 \%$ AM; $50 \%$ EC + 50\% AM; $50 \%$ EC + 50\% SV; $50 \% A M+50 \% S V)$. To evaluate the soil fauna, biological characteristics were assessed in treatments with: $100 \%$ EC; $100 \%$ AM; $100 \% \mathrm{SV} ; 50 \% \mathrm{EC}: 50 \% \mathrm{SV}$; and $50 \% \mathrm{AM}: 50 \% \mathrm{SV}$. Forty-eight months after planting, the height $(H)$ and diameter at breast height $(D B H)$ of the species $E C$ and $A M$ were measured, and estimates of basal area (BA) and wood volume with bark per tree (WBT). The abundance and diversity of the soil fauna were evaluated in leaf litter and soil (0.0-0.05 m). When intercropped with legumes, the DBH, BA and WBT of EC were higher. In contrast, for AM these variables were unaltered when intercropped with EC and SV. Plantations of EC-SV consortium promoted higher total abundance of organisms and highest values of Shannon diversity and evenness indices, especially with regard to the litter compartment.
\end{abstract}

Index terms: Eucalyptus camaldulensis, Acacia mangium, dendrometry.

\section{INTRODUÇÃO}

A exploração mineral (extração de argila), na região norte-fluminense, vem ocasionando impactos negativos ao ambiente, onde, além de promover alteração da paisagem, causa a degradação do solo e reduz a viabilidade de recuperação ambiental, por deixar o solo com vários aspectos restritivos ao crescimento de uma nova biota (Silva et al., 2009). Como alternativa para a recuperação desses ambientes, o que tem sido empregado nessa região, é o consórcio de eucalipto com espécies de leguminosas fixadoras de nitrogênio (Mendonça et al., 2008; Santiago et al., 2009; Schiavo et al., 2010), que além de se adaptarem bem a tais ambientes degradados (Franco \& Faria, 1997) podem influenciar positivamente a qualidade dos solos (Batista et al., 2008; Silva et al., 2012).

Os plantios consorciados, de modo geral, têm grande potencial para aumentar a fertilidade do solo, a produção de biomassa, a ciclagem de nutrientes e a fixação de carbono (Binkley et al., 1992; Montagnini, 2000; Balieiro et al., 2002; Resh et al., 2002; Forrester et al., 2006a; Kelty, 2006; Laclau et al., 2008). No entanto, informações sobre o efeito de plantios consorciados de eucalipto e leguminosas sobre a dinâmica de organismos do solo ainda são escassas (Batista et al., 2008; Silva et al., 2012; Bini et al., 2013), principalmente no que se refere à fauna edáfica.
A fauna do solo está intimamente relacionada à ciclagem de nutrientes (Dupont et al., 2009; Yang \& Chen, 2009; Carrillo et al., 2011), podendo ser influenciada tanto pela quantidade quanto pela qualidade de material vegetal aportado ao solo (Dupont et al., 2009; Gatiboni et al., 2009; Tripathi et al., 2010). Além disso, de acordo com Albuquerque et al. (2009), a diversidade desses organismos está associada com a grande variedade de recursos e micro-habitats que o sistema solo-serapilheira oferece, uma mistura de fases aquáticas e aéreas, o que proporciona um mosaico de condições microclimáticas, favorecendo, portanto, grande número de grupos funcionais associados.

Alguns estudos têm avaliado a ligação entre a biodiversidade acima e abaixo do solo, encontrando padrões diferenciados quanto a esse aspecto (Vanbergen et al., 2007; Wu et al., 2011). Wu et al. (2011) encontraram correlações negativas entre a diversidade de plantas e a da fauna do solo, enquanto em trabalho realizado por Vanbergen et al. (2007) a correlação foi positiva. Assim, plantios consorciados podem promover maior diversidade desses organismos no solo, tendo em vista a maior variedade de espécies vegetais cultivadas, o que proporciona maior variedade de recursos alimentares (serapilheira), com qualidade nutricional variada, formando diferentes nichos, o que facilita a coexistência de diferentes grupos taxonômicos (De Deyn et al., 2004; Bardgett et al., 2005; Vanbergen et al., 2007). 
Além de influenciar as propriedades do solo, os sistemas de plantio consorciados podem interferir no desenvolvimento das espécies arbóreas (Balieiro et al., 2002; Bauhus et al., 2004; Forrester et al., 2004, 2005, 2010; Laclau et al., 2008; Silva et al., 2012; Bini et al., 2013). Diversos estudos têm evidenciado que as interações (competição, redução competitiva e facilitação) (Forrester et al., 2005, 2006b) existentes entre as espécies nesse tipo de plantio permitem que essas apresentem resultados positivos ou negativos em seu desenvolvimento. Hunt et al. (2006), por exemplo, observaram que o consórcio de Eucalyptus nitens com Acacia dealbata promoveu maior competição por luz (avaliada pela relação altura:diâmetro; quanto mais alto o valor, maior a competição) pelo eucalipto, quando em consórcio, do que quando em monocultivo. No entanto, em estudos realizados por Forrester et al. (2004), tanto o diâmetro quanto o volume de madeira de Eucalyptus globulus foram significativamente maiores em consórcio com Acacia mearnsii do que em monocultivo, tendo o mesmo padrão ocorrido para a acácia (facilitação e redução competitiva).

De acordo com Viera et al. (2011), é difícil predizer a combinação de espécies e sítios que poderiam apresentar benefícios ou restrições ao crescimento, em razão das características intrínsecas que cada espécie apresenta, nos diferentes sítios.

Diante do exposto, este trabalho teve por objetivo avaliar a influência de sistemas de plantios (puros e consorciados) de Acacia mangium (AM), Sesbania virgata (SV) e Eucalyptus camaldulensis (EC) sobre o desenvolvimento das plantas em estudo (atributos dendrométricos) e especificamente sobre a fauna da serapilheira e dos primeiros $5 \mathrm{~cm}$ do solo.

\section{MATERIAL E MÉTODOS}

\section{Descrição da área em estudo}

O trabalho foi realizado em uma cava de extração de argila pertencente à cerâmica Stilbe Ltda, localizada no distrito de Poço Gordo ( $21^{\circ} 50^{\prime} 28,5^{\prime \prime} \mathrm{S}$; $\left.41^{\circ} 14^{\prime} 31,4^{\prime \prime} \mathrm{W}\right)$, município de Campos dos Goytacazes, RJ. O clima da região norte-fluminense é classificado de acordo com Köppen do tipo Aw, tropical quente e úmido, com período seco no inverno e chuvoso no verão, e com precipitação pluvial anual em torno de $1.020 \mathrm{~mm}$. As médias mensais de temperatura e precipitação da área em estudo, registradas no período de maio de 2006 a junho de 2007 foram de $24,1{ }^{\circ} \mathrm{C}$ e $86,6 \mathrm{~mm}$, respectivamente.

O solo original da área da cava em estudo é um Cambissolo Háplico sódico gleico, com profundidade de aproximadamente $3 \mathrm{~m}$, onde a camada superficial com maior teor de matéria orgânica foi retirada e devolvida ao fundo da cava, após a extração da argila. A cava foi nivelada mecanicamente e mantida sob pousio durante dois anos, surgindo como vegetação espontânea a braquiária [Brachiaria mutica (Forsk.) Stapf.] (Schiavo, 2005).

Após o período de pousio, realizou-se o preparo da área com uma aração e duas gradagens. A cava foi revegetada, em agosto de 2002, com Acacia mangium (AM), Eucalyptus camaldulensis (EC) e Sesbania virgata (SV), em plantios puros e consorciados (Schiavo, 2005), utilizando-se um espaçamento de $2 \mathrm{~m}$ entre plantas e $3 \mathrm{~m}$ entrelinhas. Realizou-se uma adubação nas covas com fosfato de rocha Araxá, sendo a dose aplicada equivalente a $100 \mathrm{mg} \mathrm{kg}^{-1} \mathrm{de}_{2} \mathrm{O}_{5}$.

Foi realizado um experimento, cujo delineamento utilizado foi o de blocos ao acaso com seis tratamentos e três repetições, sendo os tratamentos utilizados para avaliação dos atributos biológicos das espécies vegetais: plantios puros de EC (100EC, $100 \%$ EC) e AM (100AM, $100 \%$ AM); consórcios de 50EC:50AM (50\% EC + $50 \%$ AM); 50EC:50SV (50 \% EC + $50 \%$ SV); e 50AM:50SV ( $50 \%$ AM + $50 \%$ SV). Para avaliação dos atributos biológicos do solo, os tratamentos foram: 100EC, 100AM, 100SV (100 \% SV), 50EC:50SV e 50AM:50SV. Assim, para cada atributo avaliado foram utilizados cinco tratamentos. Entre os plantios foram alocadas duas linhas de plantas de EC, que constituíram a bordadura. A parcela experimental foi constituída por 16 plantas.

$\mathrm{Na}$ fase de produção de mudas, as espécies foram inoculadas com fungos micorrízicos arbusculares (Glomus macrocarpum, Glomus etunicatum e Entrophospora colombiana) isolados de uma área de extração de argila, pertencente à cerâmica Caco Manga Ltda., localizada no distrito de Ururaí, no município de Campos dos Goytacazes, RJ. O isolado, pertencente ao banco de inóculo do laboratório de solos da UENF, foi multiplicado em plantas de Urochoa bryzantha, em mistura de solo mais areia na proporção de 1:2 (v:v). Além dos FMAs, as espécies $A$. mangium e $S$. virgata foram inoculadas (na semente) com estirpe específica de rizóbio, sendo BR 3609, BR 6009 para AM e BR 5401 para SV, provenientes da Embrapa Agrobiologia, Seropédica, RJ.

\section{Análise da fauna edáfica}

Para avaliação da fauna edáfica, em setembro de 2006 (48 meses após o plantio), foram coletadas duas amostras por parcela, na entrelinha dos plantios, utilizando-se um gabarito de madeira de formato quadrangular de $25 \mathrm{~cm}$ de lado, que delimitou a área amostrada. Cada amostra foi dividida em subamostras de serapilheira e solo superficial $(0-5 \mathrm{~cm})$.

Para separação da fauna edáfica, no dia seguinte à coleta, as amostras foram dispostas em uma bateria de extratores do tipo Berlese-Tüllgren (Garay, 1989) modificado, pertencente ao laboratório de Fauna de Solo da Embrapa Agrobiologia. Após o período de extração (15 dias), essas foram triadas com o auxílio de microscópio estereoscópio e os organismos foram separados em grandes grupos taxonômicos. 
A partir dos resultados obtidos, foram calculados a abundância (número de indivíduo por $\mathrm{m}^{2}$ ), os índices de diversidade de Shannon (Hendrix et al., 1995) e de equitabilidade de Pielou (Pielou, 1966) de cada tratamento e a distribuição relativa de cada grupo taxonômico.

O Índice de Diversidade de Shannon $(\mathrm{H})$, que considera a riqueza das espécies e sua abundância relativa (Moço et al., 2005), é calculado pela fórmula: $\mathrm{H}=-\Sigma$ pi log pi, em que pi $=$ ni/N; ni = valor de importância de cada espécie ou grupo; e $\mathrm{N}=$ total dos valores de importância.

O Índice de Uniformidade de Pielou (e) é um índice de equitabilidade ou uniformidade, em que a uniformidade está relacionada ao padrão de distribuição dos indivíduos entre as espécies (Moço et al., 2005), sendo definido por: $\mathrm{e}=\mathrm{H} / \log \mathrm{S}$, em que $\mathrm{H}=$ Índice de Shannon; e $\mathrm{S}=$ Número de espécies ou grupos.

\section{Análises dos atributos dendrométricos das espécies vegetais}

Aos 48 meses após o plantio das espécies, foram feitas medições da altura total e circunferência à altura do peito (CAP) de todas as árvores de eucalipto e acácia, presentes em cada parcela. A altura das plantas foi medida com o auxílio de um hypsômetro (vertex III) e o CAP por meio de uma fita métrica, em que os dados foram convertidos para diâmetro à altura do peito $(\mathrm{DAP}=\mathrm{CAP} / \pi)$. Foi calculada a relação altura:diâmetro e estimados a área basal $[\mathrm{AB}=\pi(\mathrm{DAP} / 4)]$ e o volume de madeira, por planta, de todos os indivíduos presentes na parcela. $\mathrm{O}$ volume foi estimado por meio da expressão $\mathrm{V}=\left[\left(\pi^{*} \mathrm{DAP}^{2}\right) / 4\right]^{*} \mathrm{H}^{*}$, em que $\mathrm{V}=$ volume, $\mathrm{em} \mathrm{m}^{3} ; \pi=$ constante $(3,141592)$; $\mathrm{DAP}=$ diâmetro à altura do peito, em $\mathrm{m} ; \mathrm{H}=$ altura total das árvores, em $\mathrm{m}$; e $\mathrm{f}=$ fator de forma para o eucalipto $(0,5)$ e para a acácia $(0,47)$.

\section{Análise dos dados}

Os dados foram submetidos ao teste de Cochran, para verificação da hipótese de homogeneidade de distribuição (homocedastia) das variâncias dos erros amostrais (Snedecor \& Cochran, 1989), e ao teste de Lilliefors, para avaliação da distribuição normal dos resíduos (Campos, 1979). Constatada a homogeneidade e normalidade, os dados foram submetidos à análise de variância e ao teste de médias $\operatorname{Scott}-\operatorname{Knott}(\alpha=0,05)$. A análise de componentes principais (ACP) foi realizada por meio do programa PAST (Hammer et al., 2004), com as seguintes variáveis: grupos taxonômicos da fauna do solo, carbono orgânico total (COT) (Yeomans \& Bremner, 1998), nitrogênio total (NT) (CHN/S Analyser-Perkyn Elmer modelo PE 2400-II), relação C:N e aporte de serapilheira (AS) (período de um ano). Essa análise foi utilizada para reduzir as dimensões dos dados e, consequentemente, facilitar a análise por meio do gráfico do círculo de correlação (Pearson) (Herlihy \& McCarthy, 2006).

\section{RESULTADOS E DISCUSSÃO}

\section{Fauna edáfica}

A abundância da fauna edáfica entre plantios puros e consorciados apresentou padrão diferenciado, dependendo da espécie avaliada (Quadro 1).Para solos sob plantios de $S$. virgata, tanto em consórcio com a $A$. mangium (50AM:50SV) quanto com o $E$. camaldulensis (50EC:50SV), houve aumento na abundância total (AT) de organismos em relação ao plantio puro (Quadro 2), da mesma forma que nos plantios de E. camaldulensis, tendo em vista que o consórcio com a S. virgata proporcionou aumentos na AT, em relação ao plantio puro (Quadro 1). Entretanto, em solos sob a $A$. mangium, não houve diferença entre os sistemas de plantio (Quadro 1).

A maior abundância da fauna edáfica em plantios consorciados em relação a plantios puros pode estar relacionada à serapilheira com composição química diversificada (Tripathi et al., 2010). A mistura de serapilheira das espécies, com qualidade de recursos e estrutura das folhas diferentes, altera o ambiente química e fisicamente, o que modifica a superfície total de serapilheira, onde a decomposição está ocorrendo (Hector et al., 2000).

Observou-se pelo índice de Shannon que o consórcio 50EC:50SV aumentou em $63 \%$ a diversidade de organismos da fauna na serapilheira, quando comparado ao plantio puro 100EC (Quadro 1). Da mesma forma, no consórcio 50AM:50SV, em relação ao plantio puro 100AM, tanto no compartimento serapilheira quanto no solo, os aumentos foram de 35 e $49 \%$, respectivamente (Quadro 1). A qualidade nutricional da espécie $S$. virgata pode ser uma explicação para a maior diversidade $(\mathrm{H})$ da fauna do solo nos consórcios (50EC:50SV e 50AM:50SV), tendo em vista a possível melhora na qualidade da serapilheira desses ambientes.

Schiavo et al. (2009) e Silva (2009) avaliaram a qualidade nutricional de folhas jovens de $S$. virgata (sete meses de idade) e de folhas depositadas por essa espécie (48 meses de idade), respectivamente, nesse mesmo experimento. Esses autores observaram que a qualidade nutricional de $S$. virgata é superior às do E. camaldulensis e A. mangium, apresentando maiores teores de $\mathrm{N}$ e $\mathrm{P}$ e menores relações C:N e C:P. Segundo Franco \& Balieiro (1999), espécies de leguminosas arbóreas são responsáveis pela incorporação ao solo de serapilheira com relação C:N estreita, o que favorece o retorno da vida ao solo e intensifica a ciclagem de nutrientes.

O índice de Pielou também variou entre os sistemas de plantio, tanto no solo quanto na serapilheira. A maior uniformidade entre os organismos da fauna foi observada nos consórcios 50EC:50SV e 50AM:50SV, quando comparados aos plantios de 100EC e 100AM (Quadro 1). O aumento da heterogeneidade da serapilheira no solo e a formação de diferentes micro- 
habitats podem também ser justificativos para ocorrência de maiores índices de diversidade e uniformidade nos consórcios, tendo em vista a maior diversidade de plantas nesse sistema de plantio (Hooper et al., 2000; Coleman et al., 2004).

Os grupos Isopoda, Formicidae, Coleoptera, Diptera, Heteroptera, Collembola e Isoptera ocorreram sob todos os sistemas de plantio (Figura 1). O grupo Formicidae apresentou alta frequência de ocorrência nos plantios 100AM e 100EC, 34 e $48 \%$, respectivamente, enquanto os consórcios 50EC:50SV e 50AM:50SV apresentaram em torno de $17 \%$ (Figura 1). A maior proporção de formigas nos plantios puros não significa que esses sejam ambientes com estrutura de comunidade mais complexa (Silva et al., 2006). De acordo com Bruyn (1999), as formigas se adaptam facilmente às condições locais, podendo haver predomínio de uma ou mais espécies. Autores como Wink et al. (2005), Parr et al. (2007) e Alves et al. (2008) têm relatado que alta frequência de Formicidae pode ser resultado de áreas mal manejadas, o que é fundamental em estudos de impacto ambiental.
O grupo Isopoda foi tão frequente quanto o Formicidae, em todos os plantios (Figura 1), apresentando-se em maior proporção no plantio 100EC, em relação ao 50EC:50SV. O grupo Collembola, entretanto, apresentou-se com maior proporção nos consórcios, quando comparado aos plantios puros (Figura 1). Rovedder et al. (2008) destacaram esses organismos como eficientes bioindicadores da qualidade do solo. De acordo com Mussury et al. (2002) e Fernandes et al. (2009), a população e a diversidade desses organismos tendem a elevar com o aumento no teor de matéria orgânica.

O grupo classificado como "Outros" foi representado por 16 grupos taxonômicos com frequência inferior a $2 \%$. Nos plantios $100 \mathrm{SV}$ e 50EC:50SV, verificou-se elevada proporção dessa categoria, sendo encontrados valores próximos aos detectados para Formicidae e Isopoda (Quadro 2, Figura 1). Foram observados os grupos Thysanoptera, Psocoptera, Diplopoda, larva de Coleoptera e Auchenorryncha, sob todos os plantios. O grupo Symphyla foi observado apenas nos plantios 100AM, 100SV e 50EC:50SV (Quadro 3). De acordo

Quadro 1. Abundância total (AT), Índice de Shannon (H) e Índice de Pielou (e) de organismos da fauna do solo, em plantios puros e consorciados de Eucalyptus camalsulensis (EC) e Acacia mangium (AM) com Sesbania virgata (SV), em cava de extração de argila

\begin{tabular}{|c|c|c|c|c|c|}
\hline \multirow{2}{*}{ Tratamento } & \multirow{2}{*}{ AT } & \multicolumn{2}{|c|}{$\mathbf{H}$} & \multicolumn{2}{|c|}{$\mathbf{e}$} \\
\hline & & Serapilheira & Solo & Serapilheira & Solo \\
\hline & Ind $\mathrm{m}^{-2}$ & & & & \\
\hline $100 \mathrm{EC}$ & $1120 \pm 454 \mathrm{~b}$ & 2,09 & 2,36 & 0,66 & 0,68 \\
\hline 100AM & $2029 \pm 301 \mathrm{a}$ & 2,38 & 1,51 & 0,55 & 0,40 \\
\hline $100 \mathrm{SV}$ & $1029 \pm 139 b$ & 2,90 & 2,71 & 0,70 & 0,78 \\
\hline 50EC:50SV & $1744 \pm 254 \mathrm{a}$ & 3,41 & 2,74 & 0,85 & 0,72 \\
\hline 50AM:50SV & $2229 \pm 395 \mathrm{a}$ & 3,21 & 2,25 & 0,77 & 0,65 \\
\hline
\end{tabular}

Médias seguidas de mesma letra na coluna não diferem pelo teste Scott-Knott a $5 \%$.

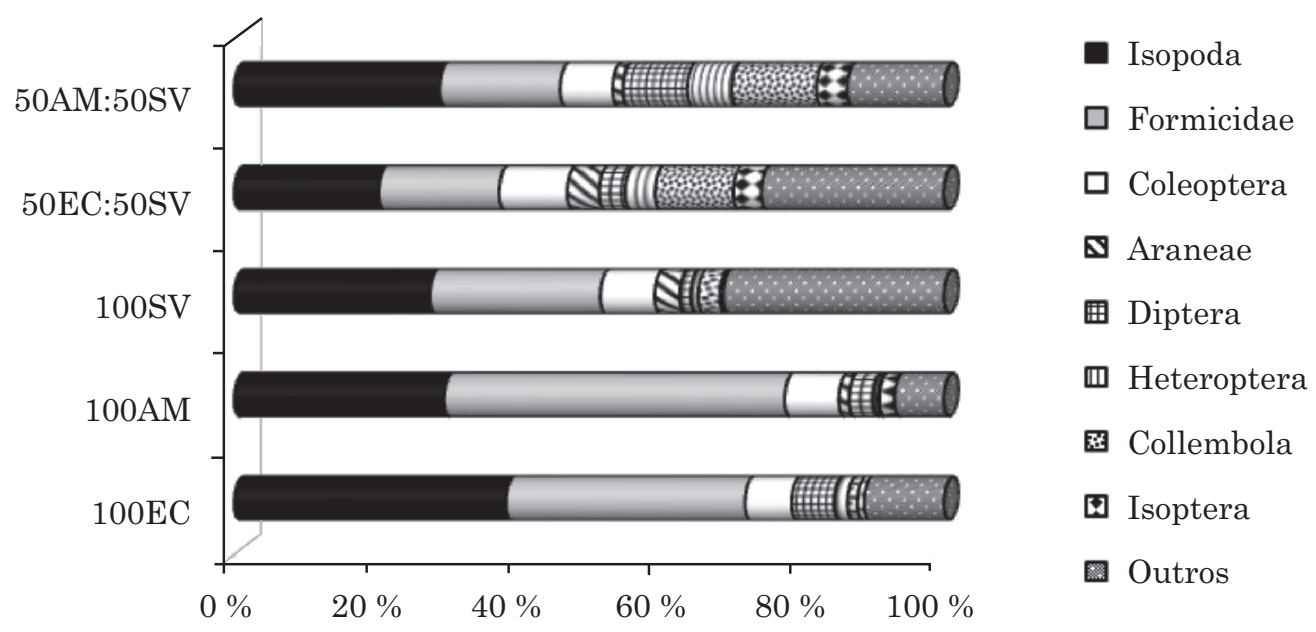

Figura 1. Distribuição relativa dos grupos taxonômicos da fauna do solo em plantios puros e consorciados de Eucalyptus camalsulensis (EC) e Acacia mangium (AM) com Sesbania virgata (SV), em cava de extração de argila. 
Quadro 2. Distribuição do grupo classificado como “Outros" em plantios puros e consorciados de Eucalyptus camalsulensis (EC) e Acacia mangium (AM) com Sesbania virgata (SV), em cava de extração de argila

\begin{tabular}{cl}
\hline Sistema de plantio & \multicolumn{1}{c}{ Grupo de fauna presente } \\
\hline 100EC & $\begin{array}{l}\text { Thysanoptera, Psocoptera, Diplopoda, Larvas de Diptera, Larvas de Coleoptera, Hymenoptera e } \\
\text { Auchenorrhyncha. }\end{array}$ \\
& Lepidoptera, Thysanoptera, Orthoptera, Psocoptera, Diplopoda, Symphyla, Pupa de Lepidoptera, \\
& Larva de Coleoptera, Larva de Lepidoptera, Larva de Trichoptera, Hymenoptera e Auchenorrhyncha. \\
& Lepidoptera, Thysanoptera, Psocoptera, Diplopoda, Symphyla, Opilionidae, Larva de Coleoptera, \\
& Larva de Trichoptera, Auchenorrhyncha e Esternorrhyncha \\
& Lepidoptera, Thysanoptera, Orthoptera, Psocoptera, Diplopoda, Symphyla, Larvas de Diptera, Larva \\
50EC:50SV & de Coleoptera, Hymenoptera e Auchenorrhyncha. \\
& Lepidoptera, Thysanoptera, Psocoptera, Diplopoda, Blattodea, Larvas de Diptera, Larva de Coleoptera, \\
& Larva de Lepidoptera, Larva de Trichoptera, Hymenoptera, Auchenorrhyncha e Esternorrhyncha \\
&
\end{tabular}

Quadro 3. Atributos dendrométricos de plantas de Eucalyptus camaldulensis (EC) e Acacia mangium (AM) em plantios puros e consorciados entre si e com Sesbania virgata (SV), em cavas de extração de argila, aos 48 meses após o plantio

\begin{tabular}{lcccccc}
\hline Tratamento & Espécie & DAP & Altura & AB & H:D & VCI \\
\hline & & $\mathrm{cm}$ & $\mathrm{m}$ & $\mathrm{m}^{2}$ & $\mathrm{~m}^{3} / \mathrm{árvore}$ \\
100EC & $\mathrm{EC}$ & $10,77 \mathrm{~B}$ & $12,55 \mathrm{~A}$ & $0,018 \mathrm{~B}$ & $1,17 \mathrm{~A}$ & $0,07 \mathrm{~B}$ \\
$100 \mathrm{AM}$ & $\mathrm{AM}$ & $16,03 \mathrm{a}$ & $16,79 \mathrm{a}$ & $0,04 \mathrm{a}$ & $1,05 \mathrm{a}$ & $0,15 \mathrm{a}$ \\
$50 \mathrm{EC}: 50 \mathrm{AM}$ & $\mathrm{EC}$ & $12,76 \mathrm{~A}$ & $16,10 \mathrm{~A}$ & $0,026 \mathrm{~A}$ & $1,26 \mathrm{~A}$ & $0,12 \mathrm{~A}$ \\
& $\mathrm{AM}$ & $15,22 \mathrm{a}$ & $13,03 \mathrm{~b}$ & $0,04 \mathrm{a}$ & $0,86 \mathrm{a}$ & $0,11 \mathrm{a}$ \\
$50 \mathrm{EC}: 50 \mathrm{SV}$ & $\mathrm{EC}$ & $13,47 \mathrm{~A}$ & $14,19 \mathrm{~A}$ & $0,028 \mathrm{~A}$ & $1,06 \mathrm{~A}$ & $0,10 \mathrm{~A}$ \\
$50 \mathrm{AM}: 50 \mathrm{SV}$ & $\mathrm{AM}$ & $15,50 \mathrm{a}$ & $14,08 \mathrm{~b}$ & $0,04 \mathrm{a}$ & $0,91 \mathrm{a}$ & $0,12 \mathrm{a}$ \\
\hline
\end{tabular}

Médias seguidas de mesma letra na coluna não diferem pelo teste Scott Knott a $5 \%$. Letras maiúsculas comparam as plantas de eucalipto em plantios puros e consorciados e letras minúsculas comparam as plantas de acácia em plantios puros e consorciados. $\mathrm{VCI}$ = volume de madeira com casca por indivíduo; $\mathrm{AB}=$ Área Basal; $\mathrm{DAP}$ = diâmetro à altura do peito; e H:D = relação altura:diâmetro.

com Souza et al. (2008) e Silva et al. (2009), esse grupo, normalmente, só é encontrado em ambientes com baixo índice de degradação.

De modo geral, os plantios não diferiram em funcionalidade dos grupos de fauna, apresentando organismos predadores, saprófagos e que exercem concomitantemente essas duas funções. $\mathrm{O}$ único grupo que esteve presente e que tem, também, como hábito alimentar a fitofagia foi o Collembola, encontrado em todos os plantios.

Como ferramenta para a distinção dos sistemas de plantios avaliados, foram geradas duas componentes principais (Fator 1 e Fator 2) para os grupos taxonômicos da fauna edáfica, o carbono orgânico total (COT), o nitrogênio total (NT), a relação C:N e o aporte de serapilheira (AS) (Figura 2). Observou-se que a distribuição das variáveis selecionadas apresentou variância acumulada de 78,19 \% para os eixos F1 e F2; o eixo F1 foi capaz de explicar $51,28 \%$ e o F2, 26,92 \% dessa variância (Figura 2).

$\mathrm{O}$ resultado da ACP, de modo geral, está de acordo com a abundância total e com os índices de diversidade de Shannon e de Pielou (Figura 2, Quadro 2). Ao longo do eixo F1 (principal), foi possível perceber a existência de dois agrupamentos distintos: sistema de plantio consorciado (50AM:50SV e 50EC:50SV), à direita; e sistema de plantio puro (100SV, 100EC e 100AM), à esquerda; para o agrupamento à esquerda, verificouse maior associação do grupo taxonômico Formicidae com o plantio 100AM, que estiveram fortemente correlacionados com o eixo $\mathrm{F} 2$. $\mathrm{O}$ agrupamento à direita, relacionou-se à maioria das variáveis; no entanto, pode-se observar que o plantio 50AM:50SV foi mais propício aos grupos taxonômicos Isopoda, Diptera, Coleoptera e Isoptera, estando também associado ao maior aporte de serapilheira (AS). Os grupos Heteroptera, Collembola, Aranae e "Outros" estiveram mais relacionados ao plantio 50EC:50SV, que esteve associado ao COT.

Assim, pôde-se constatar a influência positiva dos plantios consorciados sob a diversidade da fauna edáfica. Esse fato reforçou o que já havia sido relatado por Correia \& Andrade (2008) e Nunes et al. (2012), os quais ressaltaram que quanto mais diversa for a cobertura vegetal maior será a heterogeneidade da serapilheira, que apresentará maior diversidade das comunidades de fauna. Além disso, houve correlação 


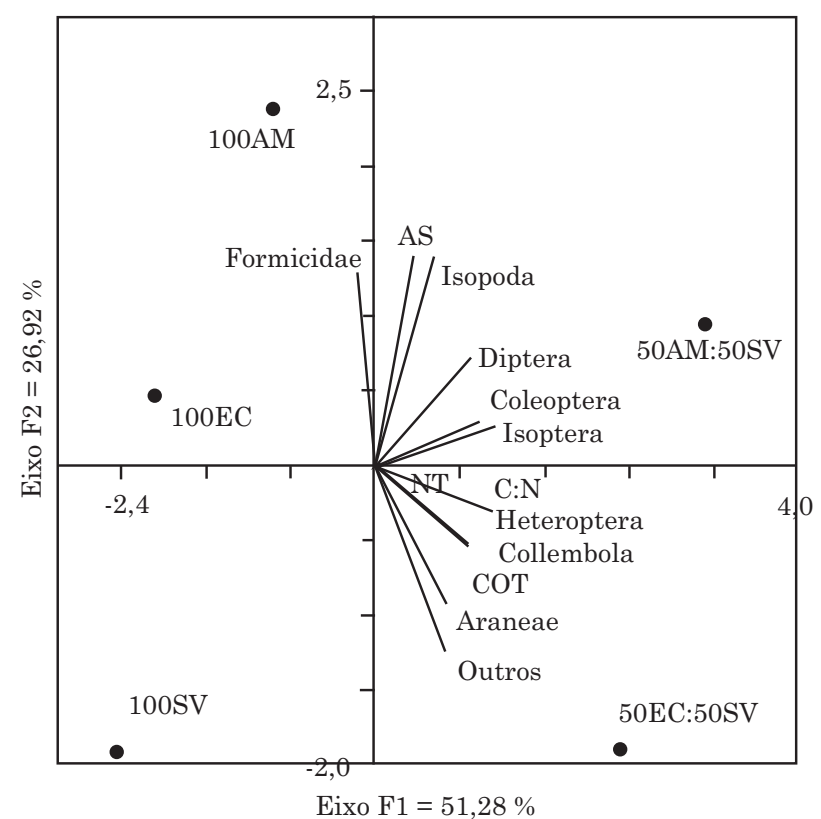

Figura 2. Análise de componentes principais (ACP) para abundância de grupos da fauna do solo, do carbono orgânico total (COT), do nitrogênio total (NT), da relação carbono:nitrogênio (C:N) e do aporte de serapilheira (AS) em plantios puros e consorciados de Eucalyptus camalsulensis (EC) e Acacia mangium (AM) com Sesbania virgata (SV), em cava de extração de argila.

desses sistemas com o aporte de serapilheira e com os teores de COT, o que pode ter contribuído para maior associação dos grupos da fauna edáfica. De acordo com Pimentel et al. (2006), o COT contribui para a atividade biológica no ambiente.

\section{Atributos dendrométricos das espécies vegetais}

Os dados de diâmetro à altura do peito (DAP), altura $(\mathrm{H})$, área basal $(\mathrm{AB})$ e volume de madeira com casca por indivíduo (VCI) das espécies $A$. mangium e $E$. camaldulensis, em plantios puros e consorciados entre si e com $S$. virgata, encontram-se no quadro 3.

Com ênfase no E. camaldulensis, verificou-se que não houve influência do consórcio no desenvolvimento em altura dessa espécie, quando comparado ao plantio puro. Entretanto, observou-se que o consórcio dessa espécie tanto com a A. mangium (50EC:50AM) quanto com a S. virgata (50EC:50SV) proporcionou incremento no DAP (18 e $25 \%$, respectivamente), AB (44 e $55 \%$, respectivamente) e VCI (71 e $57 \%$, respectivamente). Resultados similares foram obtidos por Forrester et al. (2004), para a espécie Eucalyptus globolus consorciada com Acacia mearnsii, aos quatro anos de idade, na Austrália.

Dessa forma, verificou-se, neste trabalho, interação positiva interespecífica que favoreceu o crescimento de E. camaldulensis em DAP, AB e VCI, em plantios consorciados com A. mangium (50EC:50AM) e com S. virgata (50EC:50SV). Esse padrão pode estar relacionado ao fornecimento de $\mathrm{N}$ e à ciclagem de nutrientes promovida pelas leguminosas fixadoras de $\mathrm{N}_{2}$ atmosférico, tendo em vista a redução da relação C:N no material depositado ao solo, estimulando a ação dos microrganismos decompositores e a consequente liberação de nutrientes (Khanna, 1997; Vezzani et al., 2001).

No que se refere aos plantios de $A$. mangium, não houve influência do tipo de cultivo no DAP, AB e VCI. Entretanto, a espécie em plantio consorciado apresentou menor desenvolvimento em altura, sendo a redução em torno de $20 \%$, quando comparada ao seu plantio puro. Esses dados são corroborados pelos de Laclau et al. (2008), em plantios puros e consorciados de A. mangium e Eucalyptus grandis.

Não houve influência do tipo de cultivo na relação H:D (altura:diâmetro) das árvores de E. camaldulensis e $A$. mangium. Esse padrão indica que não ocorreu competição por luz pelas árvores, quando em plantios puros ou consorciados. De acordo com Bauhus et al. (2000), quando ocorre esse tipo de competição, as árvores, com o objetivo de manter sua posição no dossel, tendem a alocar mais carbono para crescimento em altura do que em diâmetro, o que faz com que haja aumento na relação $\mathrm{H}: \mathrm{D}$.

\section{CONCLUSÕES}

1. Os sistemas de plantios consorciados de Sesbania virgata com Acacia mangium e o Eucalyptus camaldulensis, de modo geral, aumentaram a abundância total (solo + serapilheira) de organismos da fauna do solo em cavas de extração de argila, quando comparados aos plantios puros (S. virgata; $E$. camaldulensis). Além disso, apresentaram maior índice de diversidade de Shannon e equitabilidade de Pielou, principalmente no que se refere ao compartimento serapilheira.

2. Os plantios consorciados de Eucalyptus camaldulensis com as leguminosas Acacia mangium e a Sesbania virgata, em cavas de extração de argila, apresentaram-se como alternativa viável, com ganho em produtividade, tendo em vista que, até os quatro anos de idade, o desenvolvimento em diâmetro à altura do peito, área basal e volume com casca por indivíduo do E. camaldulensis são influenciados positivamente pela presença das espécies fixadoras de $\mathrm{N}_{2}$ atmosférico.

\section{AGRADECIMENTOS}

À Universidade Estadual do Norte Fluminense Darcy Ribeiro, à Embrapa Agrobiologia, e à Cerâmica Stilbe Ltda. 


\section{LITERATURA CITADA}

ALBUQUERQUE, M.P.; MACHADO, A.M.B.; MACHADO, A.F.; VICTORIA, F.C. \& MORSELLI, T.B.G.A. Fauna edáfica em sistema de plantio homogêneo, sistema agroflorestal e em mata nativa em dois municípios do Rio Grande do Sul, Brasil. Biociências, 17:59-66, 2009.

ALVES, M.V.; SANTOS, J.C.P.; GOIS, D.T.; ALBERTON, J.V. \& BARETTA, D. Macrofauna do solo influenciada pelo uso de fertilizantes químicos e dejetos de suínos no Oeste do Estado de Santa Catarina. R. Bras. Ci. Solo, 32:589$598,2008$.

BALIEIRO, F.C.; FONTES, R.L.F.; DIAS, L.E.; FRANCO, A.A.; CAMPELLO, E.F.C. \& FARIA, S.M. Accumulation and distribution of aboveground biomass and nutrients in pure and mixed stands of guachapele and Eucalyptus. J. Plant. Nutr., 25:2639-2654, 2002.

BARDGETT, R.D.; BOWMAN, W.B.; KAUFMANN, R. \& SCHMITD, T.S.K. A temporal approach to linking aboveground and belowg round ecology. Trends Ecol. Evol., 20:634-641, 2005.

BATISTA, Q.R.; FREITAS, M.S.M.; MARTINS, M.A.; SILVA, C.F. Bioqualidade de área degradada pela extração de argila, revegetada com Eucalyptus spp. e sabiá. Caatinga, 21:169-178, 2008.

BAUHUS, J.; KHANNA, P.K. \& MENDEN, N. Aboveground and belowground interactions in mixed plantations of Eucalyptus globulus and Acacia mearnsii. Can. J. For. Res., 30:1886-1894, 2000.

BAUHUS, J.; van WINDEN, A.P. \& NICOTRA, A.B. Aboveground interactions and productivity in mixed-species plantations of Acacia mearnsii and Eucalyptus globulus. Can. J. For. Res., 34:686-694, 2004.

BINI, D.; FIGUEIREDO, A.F.; SILVA, M.C.P.; VASCONCELLOS, R.L.F. \& CARDOSO, E.J.B.N. Microbial biomass and activity in litter during the initial development of pure and mixed plantations of Eucalyptus grandis and Acacia mangium. R. Bras. Ci. Solo, 37:76-85, 2013.

BINKLEY, D.; DUNKIN, K.A; DEBELL, D.S. \& RYAN, M.G. Production and nutrient cycling in mixed plantation of Eucalyptus and Albizia in Hawaii. For. Sci., 38:393-408, 1992.

BRUYN, L.A.L. Ants as bioindicators of soil function in rural environments. Agric. Ecosyst. Environ., 74:425-441, 1999.

CAMPOS, H. Estatística experimental não-paramétrica. 3.ed. Piracicaba, ESALQ, 1979. 343p.

CARRILLO, Y.; BALL, B.A.; BRADFORD, M.A.; JORDAN, C.F. \& MOLINA, M. Soil fauna alter the effects of litter composition on nitrogen mineral soil. Soil Biol. Biochem., 43:1440-1449, 2011.

COLEMAN, D.C., CROSSLEY JR., D.A. \& HENDRIX, P.F. Fundamentals of soil ecology. 2.ed. New York, Elsevier, 2004. 205p.
CORREIA, M.E.F. \& ANDRADE, A.G. Formação de serapilheira e ciclagem de nutrientes. In: SANTOS, G.A.; SILVA, L.S.; CANELLAS, L.P. \& CAMARGO, F.O., eds. Fundamentos da matéria orgânica do solo: Ecossistemas tropicais e subtropicais. 2.ed. Porto Alegre, Metrópole, 2008. p.137-158.

DE DEYN, G.B.; RAAIJMAKERS, C.E.; VAN RUIJVEN, J.; BERRENDEES, F. \& VAN DER PUTTEN, W.H. Plant species identify and diversity effects on different trophic levels of nematodes in the soil food web. Oikos, 106:576$586,2004$.

DUPONT, S.T.; FERRIS, H. \& van HORN, M. Effects of cover crop quality and quantity on nematode-based soil food webs and nutrient cycling. Appl. Soil Ecol., 41:157167, 2009 .

FERNANDES, L.H.; NESSIMIAN, J.L. \& MENDONÇA, M.C. Structure of Poduromorpha (Collembola) communities in "restinga" environments in Brazil. Pesq. Agropec. Bras., 44:1033-1039, 2009.

FORRESTER, D.I.; THEIVEYANATHAN S, C.J.J. \& MARCAR, N.E. Enhanced water use efficiency in a mixed Eucalyptus globules and Acacia mearnsii plantation. For. Ecol. Manage., 259:1761-1770, 2010.

FORRESTER, D.I.; BAUHUS, J. \& COWIE, A.L. On the success and failure of mixed species tree plantations: lessons learned from a model system of Eucalyptus globulus and Acacia mearnsii. For. Ecol. Manage., 209:147-155, 2005.

FORRESTER, D.I.; BAUHUS, J.; COWIE, A.L. \& VANCLAY, J.K. Mixed-species plantations of Eucalyptus with nitrogen fixing trees: A review. For. Ecol. Manage., 233:211-230, 2006b.

FORRESTER, D.I.; COWIE, A.L.; BAUHUS, J.; WOOD, J.T. \& FORRESTER, R.I. Effects of changing the supply of nitrogen and phosphorus on growth and interactions between Eucalyptus globulus and Acacia mearnsii in a pot trial. Plant Soil, 280:267-277, 2006a.

FORRESTER, D.I.; BAUHUSA, J. \& KHANNA, P.K. Growth dynamics in a mixed-species plantation of Eucalyptus globulus and Acacia mearnsii. For. Ecol. Manage., 193:81-95, 2004.

FRANCO, A.A. \& BALIEIRO, F.C. Fixação biológica de nitrogênio: Uma alternativa aos fertilizantes nitrogenados. In: SIQUEIRA, J.O.; MOREIRA, F.M.S.; LOPES, A.S.; GUILHERME, L.R.G.; FAQUIN, V.; FURTINI NETO, A.E. \& CARVALHO, J.G., ed. Interrelações fertilidade, biologia do solo e nutrição de plantas. Lavras, SBCS/UFLA, 1999. p.577-596.

FRANCO, A.A. \& FARIA, S.M. The contribution of $\mathrm{N}_{2}$-fixing tree legumes to land reclamation and sustainability in the tropics. Soil Biol. Biochem., 29:897-903, 1997.

GARAY, I. Relations entre l'hétérogéinéité des litières et l'organisation dês peuplements d'arthropodes édaphiques. Paris, École Normale Supérieure, 1989. (Publications du Laboratoire de Zoologie, 35) 
GATIBONI, L.C.; COIMBRA, J.L.M.; WILDNER, L.P. \& DENARDIN, R.B.N. Modificações na fauna edáfica durante a decomposição da palhada de centeio e aveia preta, em sistema plantio direto. Biotemas, 22:45-53, 2009 .

HAMMER, O.; HARPER, D.A. \& RYAN, P.D. PAST Paleontological Statistics ver. 1.12. 2004. Disponível em: <http://www.folk.uio.no/ohammer/past>. Acesso em: 13 out. 2012.

HECTOR, A.; BEALE, A.J. \& MINNS, A. Consequences of the reduction of plant diversity for litter decomposition: Effects through litter quality and microenvironment. Oikos, 90:357-371, 2000.

HENDRIX, W.; GUO, B.Z. \& NA, Z.Q. Divergence of mycorrhizal fungal communities in crop production systems. Plant Soil, 170:131-140, 1995.

HERLIHY, M. \& McCARTHY, J. Association of soil-test phosphorus with phosphorus fractions and adsorption characteristics. Nutr. Cycling Agroecosyst., 75:79-90, 2006.

HOOPER, D.U.; BIGNELL, D.E. \& BROWN, V.K. Interactions between above- and below-ground biodiversity in terrestrial ecosystems: patterns, mechanisms and feedbacks. Bioscience 50:1049-1061, 2000.

HUNT, M.A.; BATTAGLIA, M.; DAVIDSON, N.J. \& UNWIN, G. Competition between plantation Eucalyptus nitens and Acacia dealbata weeds in north-eastern Tasmania. For. Ecol. Manage., 233:260-274, 2006.

KELTY, M.J. The role of species mixtures in plantation forestry. For. Ecol. Manage., 233:195-204, 2006.

KHANNA, P.K. Comparison of growth and nutrition of young monocultures and mixed stands of Eucalyptus lobulus and Acacia mearnsii. For. Ecol. Manage., 94:105-113, 1997.

LACLAU, J.P.; BOUILLET, J.P.; GONÇALVES, J.L.M.; SILVA, E.V.; JOURDAN, C.; CUNHA, M.C.S.; MOREIRA, M.R.; SAINT-ANDRÉ, L.; MAQUÈRE, V. NOUVELLON, Y. \& RANGER, J. Mixed-species plantations of Acacia mangium and Eucalyptus grandis in Brazil. 1. Growth dynamics and aboveground net primary production. For. Ecol. Manage., 255:3905-3917, 2008.

MENDONÇA, A.V.R.; CARNEIRO, J.G.A.; BARROSO, D.G.; SANTIAGO, A.R.; FREITAS, T.A.S. \& SOUZA, J.S. Desempenho de quatro espécies de Eucalyptus spp. em plantios puros e consorciados com sabiá (Mimosa caesalpiniaefolia Benth) em cava de extração de argila. R. Árvore, 32:395-405, 2008.

MOÇO, M.K.; GAMA-RODRIGUES, E.F.; GAMARODRIGUES, A.C. \& CORREIA, M.E.F. Caracterização da fauna edáfica em diferentes coberturas vegetais na região Norte Fluminense. R. Bras. Ci. Solo, 29:555-564, 2005.

MONTAGNINI, F. Accumulation in above-ground biomass and soil storage of mineral nutrients in pure and mixed plantations in a humid tropical lowland. For. Ecol. Manage., 134:257-270, 2000.
MUSSURY, R.M.; SCALON, S.P.Q.; SILVA, S.V.S. \& SOLIGO, V.R. Study of acari and collembola populations in four cultivation systems in Dourados. Braz. Arch. Biol. Technol., 45:257-264, 2002.

NUNES, L.A.P.L.; SILVA, D.I.B.; ARAÚJO, A.S.F.; LEITE, L.F.C. \& CORREIA, M.E.F. Caracterização da fauna edáfica em sistemas de manejo para produção de forragens no Estado do Piauí. R. Ci. Agron., 43:30-37, 2012.

PARR, C.L.; ANDERSEN, A.N.; CHASTAGNOL, A. \& DUFFAUD, C. Savanna fires increase rates and distances of seed dispersal by ants. Oecologia, 151:33-41, 2007.

PIELOU, E.C. The measurement of diversity in different types of biological collections. J. Theor. Biol., 13:131-144, 1966.

PIMENTEL, M.S.; AQUINO, A.M.; CORREIA, M.E.F.; COSTA, J.R.; RICCI, M.S.F. \& DE-POLLI, H. Atributos biológicos do solo sob manejo orgânico de cafeeiro, pastagem e floresta em região do médio Paraíba fluminense-RJ. Coffee Sci., 1:85-93, 2006.

RESH, S.C.; BINKLEY, D. \& PARROTTA, J.A. Greater soil carbon sequestration under nitrogen-fixing trees compared with Eucalyptus species. Ecosystems, 5:217$231,2002$.

ROVEDDER, A.P.; VENTURINI, S.; SPAGNOLLO, E. \& ANTONIOLLI, Z.I. Colêmbolos como indicadores biológicos em solos areníticos da região Sudoeste do Rio Grande do Sul. 2008. Disponível em: <http://www.cemacufla.com.br/trabalhospdf/trabalhos\%20voluntarios/ protoc\%2069.pdf>. Acesso em: 15 out. 2008.

SANTIAGO, A.R.; BARROSO, D.G.; MENDONÇA, A.V.R.; FREITAS, T.A.S.; LAMÔNICA, K.R. \& CARNEIRO, J.G.A. Influência do sistema de plantio sobre atributos químicos de substrato de cava de extração de argila. Floresta, 39:597-604, 2009.

SCHIAVO, J.A. Revegetação de áreas degradadas pela extração de argila, com espécies micorrizadas de Acacia mangium, Sesbania virgata e Eucalyptus camaldulensis. Campos dos Goytacazes, Universidade Estadual do Norte Fluminense Darcy Ribeiro, 2005. 117p. (Tese de Doutorado)

SCHIAVO, J.A.; MARTINS, M.A. \& RODRIGUES, L.A. Avaliação nutricional de mudas de Acacia mangium, Sesbania virgata e Eucalyptus camaldulensis inoculadas com fungos micorrízicos, em casa-de-vegetação e em cava de extração de argila. Acta Sci. Agron., 31:701-707, 2009.

SCHIAVO, J.A.; MARTINS, M.A. \& RODRIGUES, L.A.R. Crescimento de mudas de Acacia mangium, Sesbania virgata e Eucalytpus camaldulensis, inoculadas com fungos micorrízicos, em casa-de-vegetação e em cava-deextração de argila. Acta Sci. Agron., 32:171-178, 2010.

SILVA, B.M.; PEDROZA, E.S.; DE PAULO, V. \& OLIVEIRA, S. Caracterização química do solo das cavas de argila no Baixo Paraíba visando a restauração das áreas de reserva legal. SEMINÁRIO DE RECURSOS HÍDRICOS DA BACIA HIDROGRÁFICA DO PARAÍBA DO SUL: RECUPERAÇÃO DE ÁREAS DEGRADADAS, 2., Taubaté, 2009. Anais... Taubaté, Serviços Ambientais e Sustentabilidade, 2009. p.625-632. 
SILVA, C.F. Atributos químicos e biológicos em cavas de extração de argila revegetadas com eucalipto e leguminosas. Campos dos Goytacazes, Universidade Estadual do Norte Fluminense Darcy Ribeiro, 2009. 172p. (Tese de Doutorado)

SILVA, C.F.; PEREIRA, M.G.; CORREIA, M.E.F. \& SILVA, E.M.R. Fauna edáfica em áreas de agricultura tradicional no entorno do Parque Estadual da Serra do Mar em Ubatuba (SP). R. Ci.. Agrár., 52:107-115, 2009.

SILVA, C.F.; SIMÕES-ARAÚJO, J.L.; SILVA, E.M.R.; PEREIRA, M.G.; FREITAS, M.F.; SAGGIN-JÚNIOR, O.J. \& MARTINS, M.A. Fungos micorrízicos arbusculares e proteína do solo relacionada à glomalina em área degradada por extração de argila e revegetada com eucalipto e acácia. Ci. Flor., 22:749-761, 2012.

SILVA, R.F.; AQUINO, A.M.; MERCANTE, M.F. \& GUIMARÃES, M.F. Macrofauna invertebrada do solo sob diferentes sistemas de produção em Latossolo da Região do Cerrado. Pesq. Agropec. Bras., 41:697-704, 2006.

SNEDECOR, W. \& COCHRAN, W.G. Statistical methods. 8.ed. Ames, Iowa State University Press, 1989. 502p.

SOUZA, R.C.; CORREIA, M.E.F.; PEREIRA, M.G.; SILVA, E.M.R.; PAULA, R.R. \& MENEZES, L.F.T. Estrutura da comunidade da fauna edáfica em fragmentos florestais na Restinga da Marambaia, RJ. R. Bras. Ci. Agrár., 3:49$57,2008$.

TRIPATHI, G.; DEORA, R. \& SINGH, J. Biological, chemical and biochemical dynamics during litter decomposition at different depths in arable soil. J. Ecol. Nat. Environ., 2:038-051, 2010.
VANBERGEN, A.J.; WATT, A.D.; MITCHEL, R.; TRUSCOTT, A.M.; PALMER, S.C.F.; IVITS, E.; EGGLETON, P.; JONES, T.H. \& SOUSA, J.P. Scalespecific correlations between habitat heterogeneity and soil fauna diversity along a landscape structure gradient. Oecologia, 153:713-725, 2007.

VEZZANI, F.M.; TEDESCO, M.J. \& BARROS, N.F. Alterações dos nutrientes no solo e nas plantas em consórcio de eucalipto e acácia-negra. R. Bras. Ci. Solo, 25:225-231, 2001.

VIERA, M.; SCHUMACHER, M.V. \& LIBERALESSO, E. Crescimento e produtividade de povoamentos monoespecíficos e mistos de eucalipto e acácia-negra. Pesq. Agropec. Trop., 41:415-421, 2011.

WINK, C.; GUEDES, V.C.; FAGUNDES, C.K. \& ROVEDDER, A.P. Insetos edáficos como indicadores da qualidade ambiental. R. Ci. Agrovet., 4:60-71, 2005.

WU, T.; AYRESB, E.; BARDGETTC, R.D.; WALL, D.H. \& GAREYA, J.R. Molecular study of worldwide distribution and diversity of soil animals. PNAS, 108:17720-17725, 2011.

YANG, X. \& CHEN, J. Plant litter quality influences the contribution of soil fauna to litter decomposition in humid tropical forests, Southwestern China. Soil Biol. Biochem., 41:910-918, 2009.

YEOMANS, J.C. \& BREMNER, J.M. A rapid an precise method for routine determination of organic carbon in soil. Commun. Soil Sci. Plant Anal., 19:1467-1476, 1988. 